\title{
An improve RCB method based on microwave induced thermo acoustic tomography
}

\author{
Jinsong Du, Wei Wang, Jie Gao and Yang Gao ${ }^{a}$ \\ Shenyang Institute of Automation, Chinese Academy of Science, Key Laboratory on Radar System Research and \\ Application Technology of Liaoning Province, Shenyang 110179, China
}

\begin{abstract}
Thermoacoustic imaging (TAI) is a non-ionizing and non-invasive imaging method, which combines the merits of high ultrasound imaging resolution with high microwave imaging contrast. In TAI, a short non-ionizing microwave pulse irradiates tissues to induce a small temperature rise, which consequently causes thermoelastic expansion to generate TA signals. By using an image reconstruction algorithm, TAI can then recover the microwave absorption distribution inside the tissue and further distinguish abnormal areas from background normal tissues. TAI for breast cancer detection is the main purpose of this study, the basic theory of TAI was introduced at first, especially the RCB reconstruction algorithm for TAI. After that, in this dissertation, two sets of thermoacoustic imaging systems were developed, which named thermoacoustic tomography and ultra-short pulse based high resolution TAI. According to experiments and theoretical studies carried out in this dissertation, the feasibility of thermoacoustic imaging method by Robust Capon Beam-former (RCB) for breast cancer detection before its clinical investigation is fully validated. Due to high imaging performance needs for the early detection of breast cancer. Based on the advantages of TAI, the potential applications of TAI for finger joints and brain diseases diagnosing are explored, which is opening up a new field for TAI.
\end{abstract}

\section{Introduction}

Recently, there has been increasing interest in using the thermoacoustic (TA) effect for biomedical applications [1,2]. TA imaging takes advantage of the excellent resolution of ultrasound imaging while keeping the merit of high contrast and good tissue penetration of microwave[3]. The TA signal strength is proportional to the microwave energy density and microwave-to-acoustic conversion efficiency[2,4]. Further enhancement of the conversion efficiency is critical for non-invasive imaging. Extensive efforts have been made to improve the efficiency of TA conversion by using exogenous contrast agents; a significant improvement is yet to be achieved[5].

Till now, X-ray mammography is the most widely used modality for breast tumour screening in high risk groups[6], but it also results in unnecessary surgery and anxiety, along with the risks of more frequent X-ray exposure causing a small but significant increase in breast cancer induced by ionizing radiation[7]. MRI provides the good tissue structure imaging in detail but still cannot provide electromagnetic parameters or acoustic parameters distribution of breast environment[8], beside its

\footnotetext{
${ }^{a}$ Corresponding author: gaoyang@sia.cn
} 
unacceptable cost for screening purpose[1,2]. Thermoacoustic imaging (TAI) is a non-ionizing and non-invasive imaging method, which combines the merits of high ultrasound imaging resolution with high microwave imaging contrast. In TAI, a short non-ionizing microwave pulse irradiates tissues to induce a small temperature rise, which consequently causes thermoelastic expansion to generate TA signals.

In recent years, various MITAT demonstrations have been reported. In 1984, Guo et al. introduced thermodynamical process. Due to the contrast in the absorption coefficient between tumor and background medium[3,5], the consequent thermal expansion caused by heating effect induces an acoustic wave at the position of the tumour. Lin and Chan built a prototype of 2-D imaging system for biological tissue. Kruger et al. performed the thermoacoustic tomography with 434-MHz microwave in five patients with documented breast cancer to show the contrast enhancement of tumors[7,8]. The microwave field energy distribution is not yet studied in-depth. LV Wang has studied the system experimental platform and qualitative image reconstruction[4,6]. The density distribution of microwave energy has been carried out by Xing Da et al. Lou et al. recently proposed a new approach of ultra-short microwave source to enhance the induced acoustic signal, which is shown to be very helpful to increase the signal-to-noise ratio (SNR) of MITAT applications.

\section{Theory Analysis}

In the thermoelastic regime the thermoacoustic pulse waveform with be determined by both the microwave absorption as well as the pulse duration. Irradiation of the absorbing layer with a microwave pulse will result in an instantaneous heat deposition inside this layer and excites TA waves[15]. As in the case of linear acoustic system, the equation of thermoelastic sound excitation in a heat-conducting medium can be given as[2,6]

$$
\alpha=\omega \sqrt{\mu \varepsilon}\left[\frac{1}{2}\left(\sqrt{1+\frac{\sigma^{2}}{\varepsilon^{2} \omega^{2}}}-1\right)\right]
$$

Where $\omega$ is the angular frequency, $\mu$ is the permeability, $\varepsilon$ is relative permittivity, $\sigma$ is the conductivity[5,6]. From (1), it is found that the absorption coefficient $\alpha$ is related with $\varepsilon$ and $\sigma$ under the fix frequency condition[5,8].

The TA signal strength is thus decided by the microwave radiation distribution. On the other hand, the propagation process of TA is related to the tissue characteristics.

$$
\left(\nabla^{2}-\frac{1}{v_{s}^{2}} \frac{\partial^{2}}{\partial t^{2}}\right) p(r, t)=-\beta \rho \frac{\partial^{2} T\left(r^{\prime}, t^{\prime}\right)}{\partial t^{2}}
$$

where $p(\boldsymbol{r}, t)$ is the induced acoustic signal, $c_{0}$ is the speed of sound, $\rho_{0}$ is the equilibrium density, $C_{p}$ is the constant pressure heat capacity per unit mass and $\beta$ is the coefficient of thermal expansion, and $H(\boldsymbol{r}, t)$ is the heating function[5,7].

As shown in Figure 1, there are $\mathrm{N}$ ultrasonic sensors in the platform, which is used to receive the TA signal. The received signal is shown as[4,7]

$$
x(t)=s(t)+n(t)
$$

In (3), $x(t)$ is the signal from the sensors, $s(t)$ is TA signal from the sample, and $n(t)$ is noise and interference from the system. It is assumed that $s(t)$ and $n(t)$ is uncorrelated, and (4) can be deduced: 


$$
R_{x x}=R_{s s}+R_{n n}
$$

Where $R_{x x}$ is the self-covariance matrix about $x(t)$, and so on.

The key of RCB is to find out the optimal weighting vector $W[1,2]$, when the gain is constant in the desired direction $\theta$. The minization of array output energy can reduce interference and enhance the SNR by the adaptive diagonal loading method. $\xi$ is the error constant [3,4].

$$
\min _{a} a^{*} R^{-1} a \quad \text { subject to }\|a-\bar{a}\|^{2}=\xi
$$

In $(5), \bar{a}=[1,1 \ldots 1]$ is the direction vector preseted. Through Lagrange multiplier method

$$
a=\bar{a}-(I+\lambda R)^{-1} \bar{a}
$$

Where $I$ is the unit diagonal matrix, and $\lambda$ can be calculated ${ }^{[6,8]}$.

$$
\left\|(I-\lambda R)^{-1} \bar{a}\right\|^{2}=\xi
$$

$\lambda \geq 0$ is the monotone decreasing function, which can be deduced by newton iteration or bisection method. From the equation (6), $a$ and the final result can be calculated.

$$
\hat{W}=\frac{R_{x x}^{-1} a(\theta)}{a(\theta) R_{x x}^{-1} a(\theta)}
$$

This paper has presented an improvement RCB method, which can achieve dynamic range path variation, and combine the synthetic aperture image and self-adaption beam-former. Comparing with the traditional TA imaging algorithm, the method can enhance SNR and increase the image resolution.

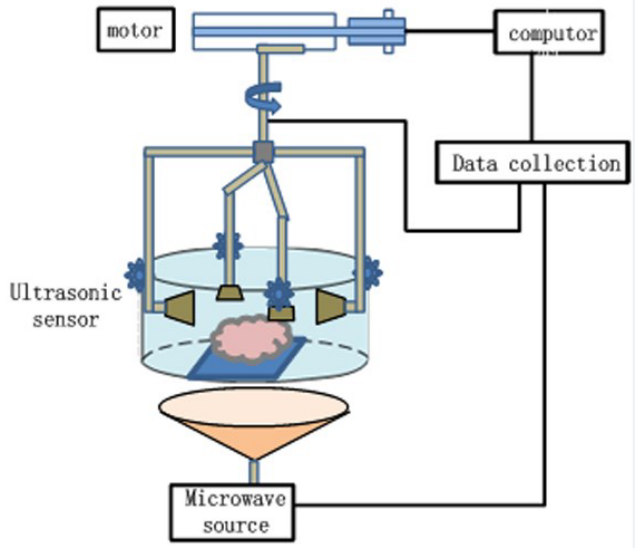

Figure 1.MITAT imaging system.

\section{Experimental Research}

In order to verify the imaging performance of the TA system, and evaluate the image reconstruction method, we used the analogy absorber as the sample which is similar with true biological tissue in the physical characteristics. The absorber is composed of agar, water and salt which were mixed by a certain percentage. Figure 2 shows TA image reconstruction result for the signal sample. In the figure, coinciding with the actual size of the absorber, the reconstruction size of the sample is $5 \mathrm{~mm} \times 5 \mathrm{~mm}$. (a) 
used the traditional Daley and Sum, and (b) did the improved RCB. From the results comparison, the result had the better SNR and resolution by improved RCB.
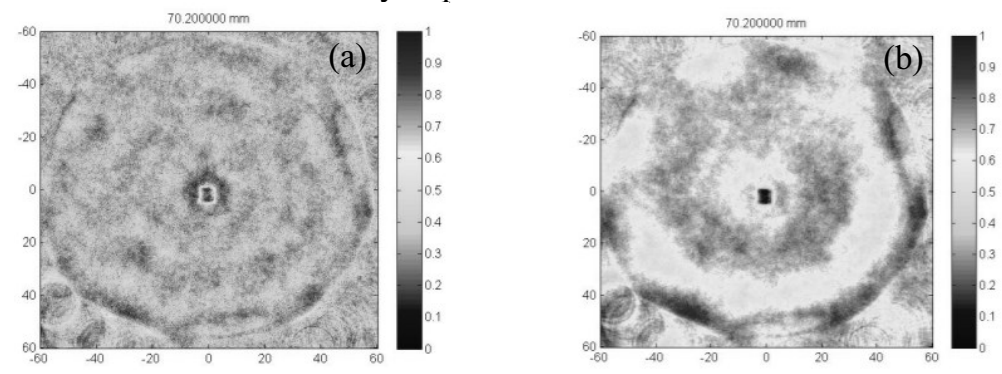

Figure 2. Recovered TA images for single absorbers.

Figure 3 showed the TA reconstruction result of both absorbers with the same physical characteristics. The distance of the both samples is $3 \mathrm{~mm}$. (a) used the traditional Daley and Sum method, and (b) did the improved RCB method. From the figure (b), the image edge is clearer, and has a better resolution and SNR. It is import to introduce the MITAT technology into the breast cancer clinical detection.
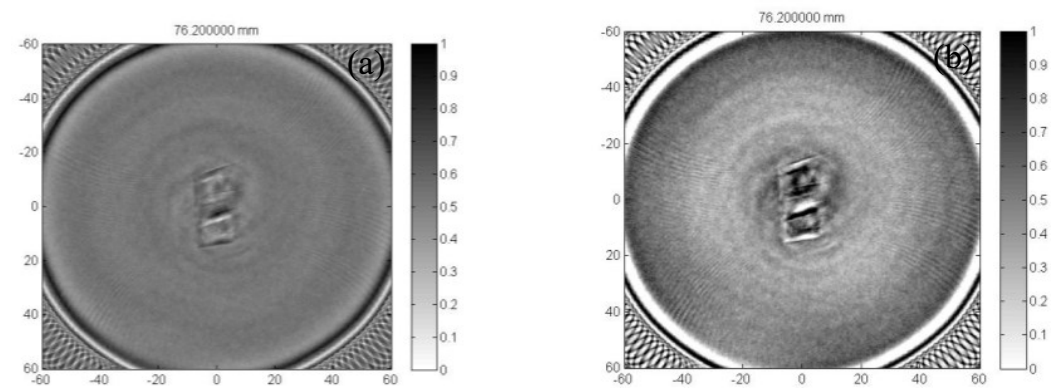

Figure 3. Recovered TA images for single absorbers.

\section{Results and Discussion}

TA signal is the ultrasound which can reflect the energy absorption distribution of biological tissue, and the reconstruction algorithm is important for reconstructing the internal structure of tissue. This paper has presented an improvement RCB method, which can achieve dynamic range path variation, and combine the synthetic aperture image and self-adaption beam-former. Comparing with the traditional TA imaging algorithm, the method can enhance SNR and increase the image resolution. Combine the theoretical analysis and experimental data, which comes from the S-band microwave induced thermo-acoustic imaging system, we have demonstrated that the image algorithm is important.

\section{References}

1. Cunguang Lou, Sihua Yang, Zhong Ji, Qun Chen, and Da Xing. Ultra-short Microwave-Induced Thermoacoustic Imaging: A Breakthrough in Excitation Efficiency and Spatial Resolution. PHYSICAL REVIEW LETTERS, pp. 208101-1 208101-5 (2012).

2. Pramanikm KUG, Changhui Li, et al. Designand evaluation of a novel breast cancer detection system combining both thermoacoustic andphotoacoustic (PA) tomography[J]. Medical Physics, 35( 6) :2218-2223 (2008).

3. Nie Liming, Zhou Quan, Yang Diwu. Microwave-induced thermoacoustic scanning CT for highcontrast and noninvasive breast cancer imaging[J]. Medical Physics, 35( 9): 4026-4032 (2008). 
4. KELLNBERGERS, HAJIABOLIA, RAZANSKYD, et al. Near-field thermoacoustic tomography of small animals[J]. Physics inMedicine andBiology, 56(11): 3433-3444 (2011).

5. Xin Bi, Lin Huang, Jinsong Du, Weizhi Qi, Yang Gao. Pulsed Microwave Energy Spatial Distribution Imaging by Means of Thermoacoustic Tomography, Acta Phys. Sin., 64(1), pp. 014301-1 014301-6 (2015).

6. Jinsong Du, Yang Gao, Xin Bi, Weizhi Qi, Lin Huang, Jian Rong. S-band microwave induced thermo-acoustic tomography system, Acta Phys. Sin. 64(3), pp. 034301-1 034301-6 (2015)

7. Huabei Jiang, Yong Xu, Nicusor Iftimia Julie Eggert and etc., "Three Dimensional Optical Tomographic Imaging of Breast in A Human Subject”, IEEE Trans on Med., Vol. 20, No. 12, pp: 1334-1340 (2010).

8. Zeng L M, Xing D, Gu H M, Fast microwave-induced thermoacoustic tomography based on multi-element phase-controlled focus technique. Chin Phys Lett, 23(5), pp. 12125-1218 (2006). 